\title{
Erratum to: No regrets: Young adult patients in psychiatry report positive reactions to biobank participation
}

\author{
Janet L. Cunningham*, Manuel Zanzi, Mimmie Willebrand, Lisa Ekselius and Mia Ramklint
}

\section{Erratum}

During the production of this article [1] the authors noticed that there was a mistake in Table 1 . The correct version of the table appears on the following page. The value after 'Any major depressive disorder' has been changed from 134 to 314.

Received: 30 January 2017 Accepted: 2 February 2017

Published online: 20 February 2017

\section{Reference}

1. Janet L. Cunningham Manuel Zanzi, Mimmie Willebrand, Lisa Ekselius and Mia Ramklint, No regrets: Young adult patients in psychiatry report positive reactions to biobank participation. BMC Psychiatry. 2017;17:21. doi:10.1186/ s12888-017-1199-0. 
Table 1 Characteristics of participants who chose to participate in UPP

\begin{tabular}{lll}
\hline & & $n(\%)$ \\
\hline Patients & Total (\%) & $463(100)$ \\
& Mean (range) & $21(18-25)$
\end{tabular}

Gender

$\begin{array}{ll}\text { Female } & 362(78.2) \\ \text { Male } & 101(21.8)\end{array}$

Diagnosis (current)

Any major depressive disorder

$314(67.8)$

Any bipolar disorder

$58(12.5)$

Any anxiety disorder

$278(60.0)$

Missing information

$6(1.3)$

Level of education

University

$211(45.6)$

Upper secondary school

191 (41.3)

9 year elementary school

$31(6.7)$

Missing information

$10(2.2)$

Controls

Total (\%) $105(100)$

Age

$$
22(18-30)
$$

Gender

Female $\quad 79(75.2)$

Diagnosis (current)

Any major depressive disorder

0

Any bipolar disorder

Any anxiety disorder

Missing information

$5(4.7)$

Level of Education

$\begin{array}{ll}\text { University } & 101(96.2) \\ \text { Upper secondary school } & 0 \\ 9 \text { year elementary school } & 1(0.01) \\ \text { Missing information } & 3(0.03)\end{array}$

